\title{
PESSOAS COM DEFICIÊNCIA: PERCEPÇÃO DE SEUS CUIDADORES QUANTO AO ATENDIMENTO ODONTOLÓGICO
}

\section{PEOPLE WITH DISABILITIES: PERCEPTION OF THEIR CAREGIVERS ABOUT DENTAL CARE}

\author{
Lauren Volquind ${ }^{1}$, Lucas Bozzetti Pigozzi², Mariá Cortina Bellan ${ }^{3}$, Marília Paulus ${ }^{4}$, Alexandre Conde ${ }^{5}$
}

\author{
Submetido: $17 / 11 / 2020$ \\ Aprovado: 07/12/2020
}

\section{RESUMO}

As pessoas com deficiência $(\mathrm{PcD})$ são os indivíduos que apresentam desvios de natureza física, sensorial ou intelectual, que os limite de participação integral na sociedade, por um certo período ou a vida toda. A PcD está exposta a desenvolver desordens na cavidade bucal que podem agravar suas alterações sistêmicas presentes, causando uma higiene oral negligenciada. O objetivo deste estudo é avaliar, através da percepção dos cuidadores, a qualidade de atendimento odontológico ofertado para pessoas com deficiência no município de Caxias do Sul-RS, bem como identificar a existência de conhecimento do tratamento odontológico realizado na $\operatorname{PcD}$ e a existência de acesso ao serviço odontológico para esse grupo. Como metodologia foi aplicado um questionário para pais, responsáveis legais e/ou cuidadores de pessoas com deficiência que frequentam a clínica VidaFisio, em Caxias do Sul-RS. O questionário contém perguntas dos estudos de Santos et al. (2011), que abrange questões sobre tratamento odontológico, acesso aos serviços e qualidade de atendimento. Cerca de $58,1 \%$ das pessoas entrevistadas relataram que a qualidade de atendimento é boa na cidade e $41,9 \%$ como ótima. A boa receptividade e cordialidade das clínicas odontológicas teve índices altos na pesquisa. Todos os profissionais prestaram informações sobre a importância de uma boa saúde bucal para os entrevistados. Conclui-se que foi possível avaliar a qualidade do atendimento odontológico sendo considerada boa pela maioria dos entrevistados, mas pode melhorar se houver mais profissionais disponíveis a atender pessoas com deficiência, diminuindo as listas de espera do setor público de saúde. Foi possível identificar a existência de conhecimento dos cuidadores a respeito do tratamento odontológico realizado na $P c D$ sendo considerado importante para o entendimento e manutenção de uma boa saúde bucal. Além disso, também foi constatada a existência de acesso ao serviço odontológico para o grupo pesquisado na cidade.

PALAVRAS-CHAVE: Odontologia. Saúde Bucal. Pessoas com Deficiência. Cuidadores.

\begin{abstract}
People with disabilities (PwD) are individuals who have deviations of a physical, sensory or intellectual nature, which limit them to full participation in society for a certain period or for a lifetime. $P w D$ are exposed to develop disorders in the oral cavity that can aggravate its present systemic changes, causing neglected oral hygiene. This study aims to evaluate, through the perception of the caregivers, the quality of dental care offered to people with disabilities in city of Caxias do Sul-RS, as well as to identify the existence of knowledge of dental treatment performed in PwD and the existence

\footnotetext{
${ }^{1}$ Graduanda do Curso de Graduação em Odontologia - FSG Centro Universitário - Caxias do Sul/RS - ORCID: https://orcid.org/0000-0002-5405-3752

2 Mestre em Prótese Dentária - Professor do Curso de Graduação em Odontologia - FSG Centro Universitário Caxias do Sul/RS - ORCID: https://orcid.org/0000-0003-0368-8149

3 Mestra em Materiais Dentários - Professora do Curso de Graduação em Odontologia - FSG Centro Universitário - Caxias do Sul/RS - ORCID: https://orcid.org/0000-0002-7074-3518

4 Doutora em Materiais Dentários - Professora do Curso de Graduação em Odontologia - FSG Centro Universitário - Caxias do Sul/RS - ORCID: https://orcid.org/0000-0002-2615-5284

5 Doutor em Materiais Dentários - Professor do Curso de Graduação em Odontologia - FSG Centro Universitário - Caxias do Sul/RS - ORCID: https://orcid.org/0000-0003-0224-2313
} 


\title{
RECIMA21 - REVISTA CIENTÍFICA MULTIDISCIPLINAR
}

\author{
PESSOAS COM DEFICIÊNCIA: PERCEPÇ̃̃O DE SEUS CUIDADORES QUANTO AO \\ ATENDIMENTO ODONTOLÓGICO \\ Lauren Volquind, Lucas Bozzetti Pigozzi, Mariá Cortina Bellan, Marília Paulus, Alexandre Conde
}

\begin{abstract}
of access to dental service for this group. As a methodology, a questionnaire was applied to parents, legal guardians and/or caregivers of people with disabilities who attend VidaFisio clinic, in Caxias do Sul. The questionnaire contains questions from studies by Santos et al. (2011), which are related to dental treatment, access to services and quality of care. As results, $58.1 \%$ of the people interviewed reported that the quality of care is good and $41.9 \%$ as excellent. The good reception and cordiality of dental clinics had high rates in the survey. All professionals provided information about the importance of a good oral health to the interviewees. As conclusion, it was possible to evaluate the quality of dental care, being considered good by most of the interviewees, but it can improve if there are more professionals available to treat people with disabilities, reducing waiting lists in the public health sector. It was possible to identify the existence of knowledge of caregivers regarding dental treatment performed in PwD, being considered important for the understanding and maintenance of good oral health. In addition, there was also the existence of access to dental services for the group surveyed in the city.
\end{abstract}

KEYWORDS: Dentistry. Oral Health. Disabled Persons. Caregivers.

\section{INTRODUÇÃO}

A compreensão que os indivíduos têm sobre a condição bucal é uma importante referência à qualidade de vida, que simboliza um compromisso pessoal para a obtenção de uma vida saudável e faz síntese às condições concretas de saúde, às respostas subjetivas, aos valores e às perspectivas culturais. O indivíduo acometido por alterações no estado de saúde geral preocupa-se mais com a disfunção existente, razão pela qual ele se encontra acamado, internado ou limitado e considera irrelevantes os cuidados com a saúde bucal. ${ }^{1}$

No Brasil, segundo o censo demográfico de 2010 divulgado pelo Instituto Brasileiro de Geografia e Estatística, cerca de 45.606.048 milhões de pessoas têm algum tipo de deficiência, o equivalente a $23,9 \%$ da população geral. Essa deficiência pode ser visual, auditiva, motora, mental ou intelectual. Ainda de acordo com o censo do IBGE de 2010, a deficiência mais recorrente no Brasil é a visual (18,6\%), seguida da motora (7\%), seguida da auditiva $(5,10 \%)$, e, por fim, da deficiência mental $(1,40 \%){ }^{2}$

Quando falamos de pessoas com deficiência $(\mathrm{PcD})$ estamos nos referindo ao grupo de indivíduos que apresentam desvios de natureza física, sensorial ou intelectual, que os limite de atuação integral na sociedade, seja por um certo período ou durante a vida toda. ${ }^{3}$

Para fins de assistência odontológica, o Ministério da Saúde utiliza-se do conceito de pessoas com deficiência que é:

"Todo usuário que apresenta uma ou mais limitações, temporárias ou permanentes, de ordem mental, física, sensorial, emocional, de crescimento ou médica, que o impeça de ser submetido a uma situação odontológica convencional. As razões das necessidades especiais são inúmeras e vão desde doenças hereditárias, defeitos congênitos, até as alterações que ocorrem durante a vida, como moléstias sistêmicas, alterações comportamentais, envelhecimento etc. Esse conceito é amplo e abrange, entre os diversos casos que requerem atenção diferenciada, as pessoas com deficiência visual, auditiva, física ou múltipla (conforme definidas nos Decretos 3296/99 e 5296/04) que eventualmente precisam ser submetidas à atenção odontológica especial" (Cadernos da Atenção Básica, 2008, p. 67). 


\title{
RECIMA21 - REVISTA CIENTÍFICA MULTIDISCIPLINAR
}

\author{
PESSOAS COM DEFICIÊNCIA: PERCEPÇ̃̃O DE SEUS CUIDADORES QUANTO AO \\ ATENDIMENTO ODONTOLÓGICO \\ Lauren Volquind, Lucas Bozzetti Pigozzi, Mariá Cortina Bellan, Marília Paulus, Alexandre Conde
}

A Constituição Federal, desde 1988, apresenta princípios gerais de política de inclusão de pessoas com deficiência, nela constando como "promover o bem de todos, sem preconceitos de origem, raça, sexo, cor, idade e quaisquer outras formas de discriminação". A Portaria n 599/GM, de 23 de março de 2006, que define a implantação dos Centros de Especialidades Odontológicas (CEO), estabelece que todo CEO deva realizar atendimento a PcD. ${ }^{4}$

Por apresentarem alterações sistêmicas predisponentes, como por exemplo cardiopatias, doenças reumáticas, doenças renais crônicas, as pessoas com deficiência estão expostas a desenvolver doenças na cavidade bucal que podem acentuar as disfunções sistêmicas presentes. A higiene oral negligenciada, a limitação física e/ou mental e a dieta cariogênica são fatores que estão aliados à progressão dos distúrbios bucais, sendo a cárie e a doença periodontal as mais prevalentes em PcD. ${ }^{3}$

Nesta área de atuação, o maior desafio é encontrar profissionais motivados, especializados ou mesmo interessados para atender à grande demanda existente deste grupo particular de pacientes. Há uma necessidade de estabelecer programas preventivos e curativos para PcD, tanto em rede pública quanto particular, uma vez que eles apresentam maiores índices de cárie e doença periodontal do que a população em geral. ${ }^{5}$

Constantemente os cuidadores sentem-se desmotivados por não possuírem instruções de como realizar uma higiene bucal adequada e ainda mencionam dificuldades para desempenhá-la. Essas dificuldades estão relacionadas com o manuseio desses indivíduos, visto que podem apresentar limitações motoras, comportamentos violentos e falta de interesse em colaborar. ${ }^{6}$

Para o tratamento odontológico possuir êxito, a compreensão e o engajamento tanto dos pais, cuidadores e responsáveis quanto dos pacientes no planejamento são indispensáveis. Além de uma comunicação coerente, o tratamento individualizado, a motivação, a criação de vínculos e a educação voltada para a saúde bucal também são elementos que podem solucionar as adversidades que orbitam em torno do atendimento às pessoas com deficiência. ${ }^{5}$

A atuação dos cuidadores é importante na higienização bucal das pessoas com deficiência, pois são eles que verificam quaisquer anormalidades presentes na cavidade bucal. Entretanto, grande parte dos responsáveis ainda têm pouco conhecimento quanto a educação alimentar e a influência na higiene bucal e seguem com uma oferta de produtos ricos em açúcar, o que traz uma repercussão muito forte de cárie dentária, levando os cuidadores a buscarem atenção odontológica ao sinal de dor dentária, presença de sangramento gengival, hipersalivação ou halitose. ${ }^{7}$

Atualmente, a abordagem terapêutica voltada para esse público é baseada em uma anamnese bem detalhada, no conhecimento e no envolvimento emocional do cirurgião-dentista, bem 


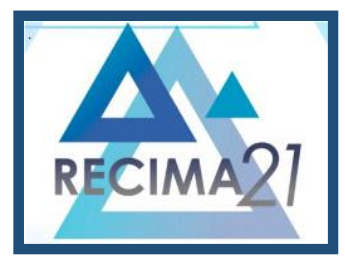

\title{
RECIMA21 - REVISTA CIENTÍFICA MULTIDISCIPLINAR
}

\author{
PESSOAS COM DEFICIÊNCIA: PERCEPÇ̃̃O DE SEUS CUIDADORES QUANTO AO \\ ATENDIMENTO ODONTOLÓGICO \\ Lauren Volquind, Lucas Bozzetti Pigozzi, Mariá Cortina Bellan, Marília Paulus, Alexandre Conde
}

como a paciência e a determinação dele. Vale realçar que a saúde bucal é um componente da saúde geral e que, caso esteja afetada, pode trazer impacto negativo para o bem-estar e na qualidade de vida. $^{8}$

Ainda não existe uma técnica padronizada para fazer uma higiene bucal adequada para esta população. Cada cuidador, juntamente com o cirurgião-dentista especializado, saberá qual será o melhor método de adequar a cavidade bucal. Durante as consultas de manutenção, o profissional identificará quais são as necessidades a serem alteradas quanto a forma de cuidados domiciliares. Escovas elétricas ou modificadas, bem como abridores de boca e fio dental em haste são instrumentos que facilitam a função do cuidador, entretanto são pouco conhecidas fora do consultório odontológico. ${ }^{9}$

A oferta de tratamento para estes pacientes ainda é escassa, pois os profissionais sentem dificuldade de técnicas específicas e de preparo psicológico para desenvolver um atendimento adequado, com isso a presente pesquisa teve como objetivo avaliar através da percepção dos cuidadores, a qualidade do atendimento odontológico ofertado para pessoas com deficiência no município de Caxias do Sul-RS.

\section{REFERENCIAL TEÓRICO}

\subsection{Pessoas com Deficiência (PcD)}

A pessoa com deficiência $(\mathrm{PcD})$ é aquele indivíduo que mostra qualquer tipo de condição que demande cuidado diferenciado por um prazo de sua vida ou continuadamente. ${ }^{9,11,13}$. Elas carecem de atenções médicas e odontológicas direcionadas à sua condição específica. Deste modo, os profissionais da saúde devem estar qualificados para ofertar um tratamento apropriado. O estado clínico da PcD está associado com a habilidade de higiene bucal, sendo que as limitações na motricidade e na cognição podem comprometer esta ação. Muitas vezes, os problemas na cavidade bucal são colocados em segundo plano por profissionais não ligados à odontologia. Portanto, quanto mais cedo é a busca por atendimento, maior é a cooperação do paciente durante o tratamento odontológico. ${ }^{8}$

Qualquer indivíduo deve ser capaz de se pronunciar, mastigar, sorrir, reconhecer os sabores, viver sem dor e desconforto e se comunicar com outras pessoas sem embaraço. Aspectos físico, social e psicológico são meios em que as pessoas notam a importância e a repercussão da saúde bucal na qualidade de vida. ${ }^{12}$ Por apresentarem alterações sistêmicas predisponentes, as pessoas com deficiência estão expostas a desenvolver doenças na cavidade bucal que podem acentuar as disfunções sistêmicas presentes. ${ }^{8}$ A higiene oral negligenciada, a limitação física e/ou mental e a dieta cariogênica são fatores que estão aliados à progressão dos distúrbios bucais, sendo a cárie e a doença periodontal as mais prevalentes na PcD. ${ }^{3}$ 


\title{
RECIMA21 - REVISTA CIENTÍFICA MULTIDISCIPLINAR
}

\author{
PESSOAS COM DEFICIÊNCIA: PERCEPÇÃO DE SEUS CUIDADORES QUANTO AO \\ ATENDIMENTO ODONTOLÓGICO \\ Lauren Volquind, Lucas Bozzetti Pigozzi, Mariá Cortina Bellan, Marília Paulus, Alexandre Conde
}

As condições bucais inadequadas da $\mathrm{PcD}$ também podem estar vinculadas com a dificuldade de receber cuidado. Alimentação pastosa, uso prolongado de mamadeiras, fármacos com alto teor de sacarose ou fármacos que podem causar xerostomia estão muitas vezes associados com a deglutição atípica, malformação dentária e oclusal, agravando as condições bucais. ${ }^{7,10}$ Elevados sinais de cárie, traumatismo dentário e más oclusões estão acometendo os pacientes. Além das já citadas, doenças gengivais, infecções do periodonto, respiração bucal e hiperplasia gengival podem ser visualizadas também. ${ }^{11}$

Nem sempre a introdução da higiene bucal é presente na vida dos pacientes e consultas preventivas para pessoas com deficiência são escassas. O conhecimento dos cuidadores tem relevância para a manutenção da saúde da $\mathrm{PcD}$, pois promove bem-estar e, logo, necessitam de maior instrução e indução por parte dos profissionais para melhorarem as circunstâncias da vida dos pacientes. ${ }^{7}$ Maiores são as chances dessas pessoas apresentarem acúmulo de placa bacteriana expressivo quando os níveis de escolaridade e socioeconômico dos responsáveis forem inferiores. ${ }^{5}$

$\mathrm{Na}$ odontologia, a PcD é classificada em nove grupos: deficiência mental e física, anomalias congênitas, alterações comportamentais, transtornos psiquiátricos, disfunções sensoriais e de comunicação, doenças crônicas do sistema, doenças infectocontagiosas, condições sistêmicas. Os fundamentos das deficiências são diversos, que pode ir desde doenças hereditárias e deformidades congênitas, até as alterações que sucedem por toda a vida, como doenças sistêmicas, alterações de comportamento, envelhecimento, entre outros. ${ }^{12}$

Já é constatado que a saúde bucal influencia na qualidade da saúde geral, e que indivíduos com deficiência têm mais risco de desordens na cavidade bucal. ${ }^{3,13}$ Por isso, as orientações sobre manejo, controle de biofilme e outros tópicos estão relativamente ligados à realidade desses pacientes e deve ser uma prática comum em nossa sociedade, onde a $\mathrm{PcD}$ e sua saúde bucal não podem ser menosprezadas. ${ }^{14}$

Antecedentes socioeconômicos, como idade, educação em saúde bucal, consultas odontológicas e nível de escolaridade influenciam os cuidados com a PcD. ${ }^{15}$ Por não terem acompanhamento regular, atender pessoas com deficiência tem se mostrado omisso em função da falta de preparo, experiência, habilitação e limitações específicas e inespecíficas do próprio paciente e até dos cuidadores. ${ }^{16} \mathrm{~A}$ melhora do estado de saúde bucal entre indivíduos com deficiência pode ser agrupada em três categorias: aprimorar os métodos de educação em saúde e criando um ambiente amigável para os indivíduos, melhorar a alfabetização em saúde bucal e a qualidade do atendimento entre os cuidadores e estabelecendo um curso de odontologia para dentistas que gostariam de tratar estes pacientes. ${ }^{17}$

\subsection{Direito de acessibilidade aos serviços odontológicos}




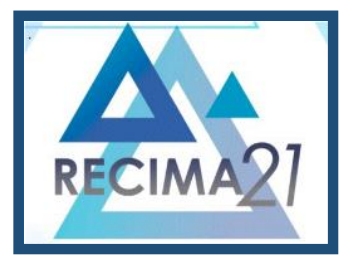

\title{
RECIMA21 - REVISTA CIENTÍFICA MULTIDISCIPLINAR
}

\author{
PESSOAS COM DEFICIÊNCIA: PERCEPÇ̃̃O DE SEUS CUIDADORES QUANTO AO \\ ATENDIMENTO ODONTOLÓGICO \\ Lauren Volquind, Lucas Bozzetti Pigozzi, Mariá Cortina Bellan, Marília Paulus, Alexandre Conde
}

Segundo o Caderno de Atenção Básica n 17 - Saúde Bucal, a pessoa com deficiência deve ter prioridade no acesso à atenção primária. Somente os casos de maior complexidade devem ser encaminhados aos Centros de Especialidades Odontológicas (CEO). ${ }^{4} \mathrm{O}$ avanço nas políticas públicas que valorizam o cidadão com respeito às suas características e especificidades se refletem também na saúde, priorizam o acesso deste público a todos os serviços oferecidos pelo Sistema Único de Saúde (SUS). ${ }^{18}$

No Brasil, as necessidades odontológicas são pouco investigadas, gerando como resultado uma higiene bucal deficiente em muitas PcDs. Em programas assistenciais de rotina, muitas vezes as pessoas com deficiência conseguem se favorecer e por razão disso, a advertência sobre doenças bucais deve ser realizada por meio de programas de educação e prevenção. Nos programas preventivos para cada tipo de enfermidade especial, o aprimoramento de técnicas de condicionamento, incentivo, precaução e promoção da saúde bucal deveriam estar listados. Entretanto, o alcance em possuir profissionais especializados e/ou apoios governamentais é pouco expressivo na sociedade atual. ${ }^{7}$

O manejo desse grupo ainda é algo muito questionado devido aos poucos profissionais interessados em complementar essa carência odontológica e a atenção maior que o cirurgiãodentista deve ter ao atendê-los. ${ }^{12}$ Há uma necessidade de aplicar recursos odontológicos preventivos e curativos para PcD porque elas apresentam maiores evidências de cárie (CPO-D) e doença periodontal do que a população geral, bem como higiene bucal deficiente e falta de acompanhamento odontológico. As políticas preventivas devem ser contínuas para que otimizem nos pais, professores e na $\mathrm{PcD}$ a prevenção e o cuidar. ${ }^{5}$ Como consequência de um atendimento com antecedência, os hábitos criados durante o tratamento tendem a perdurar por toda a vida. $O$ atendimento não é eficaz quando não há colaboração do paciente e do cuidador, como também com a falta de preparo do cirurgião-dentista. ${ }^{9}$

O atendimento das pessoas com deficiência inicia na atenção básica, que caso seja necessário, encaminhará o indivíduo para a atenção secundária ou terciária, sendo o Centro de Especialidades Odontológicas e atendimento a nível hospitalar, respectivamente. O tratamento a nível hospitalar é feito apenas em situações em que não há cooperação do paciente na cadeira odontológica. Verifica-se que há necessidade de apoio político para infraestrutura e gestão desses serviços. Com essas necessidades postas em prática será possível testemunhar um indicativo de que cada vez mais os cirurgiões-dentistas possuirão base para instruir, motivar e conscientizar esses pacientes e os cuidadores sobre a saúde bucal. ${ }^{19}$

Embora ainda existam algumas falhas na prevenção e na promoção da saúde bucal a serem adaptadas, o serviço público de saúde não deve ser modelado como uma instituição de caridade, 


\section{RECIMA21 - REVISTA CIENTÍFICA MULTIDISCIPLINAR}

PESSOAS COM DEFICIÊNCIA: PERCEPÇÃO DE SEUS CUIDADORES QUANTO AO

ATENDIMENTO ODONTOLÓGICO

Lauren Volquind, Lucas Bozzetti Pigozzi, Mariá Cortina Bellan, Marília Paulus, Alexandre Conde

mas sim como um espaço para atender a todos, como consta nos direitos dos indivíduos ${ }^{20,21} \mathrm{~A}$ demanda para atender esses pacientes é crescente e é direito fundamental de todos ter acesso aos tratamentos. Por muitas vezes apresentarem limitações, é um grupo com alto índice de vulnerabilidade a exibir patologias bucais. Trabalhar de forma integrada para atender as pessoas com deficiência, isto é, com a participação da enfermagem, medicina, fisioterapia, fonoaudiologia tem se mostrado muito eficaz para o bem-estar do sujeito. Adequar o maior número de cirurgiõesdentistas, provendo materiais e instrumentais adaptados é o que se tem como mudanças nas redes de atendimento a PcD. ${ }^{21}$

Planejar uma intervenção é um dos principais aspectos ao realizar programas de promoção de saúde. Uma maneira de quebrar barreiras pode ser prover diretrizes. Diretrizes práticas para cuidados bucais em PcD devem existir para cobrir a atual falta de protocolos e rotinas. Para acudir às necessidades atuais, a educação em saúde bucal deve ser prática e especialmente personalizada para as deficiências dos pacientes e prestadores de cuidados. ${ }^{22}$

\subsection{Qualidade do atendimento odontológico para PcD}

Fatores como a humanização no atendimento é o que tem se notado estar disponível para estes pacientes. A relação entre profissional, paciente e família/cuidador deve ser genuína para que se tenha um bom resultado de tratamento. ${ }^{23}$ Criar estratégias que realizem tratamentos de forma mais ampla, dando valor a implicação destes problemas no contexto de vida da família é uma ação que valoriza o aspecto humano. ${ }^{24}$

Conhecer as alterações do paciente, tanto pelo profissional quanto pelo cuidador é o que trará uma rotina específica e o sucesso do tratamento, assim como na prevenção de futuras doenças bucais. As técnicas modificadas para higiene bucal, embora pouco reconhecidas pelos cuidadores, são os_fatores que ajudam e facilitam na hora de manter os hábitos higiênicos. Não há um método ideal para os indivíduos, todavia já se sabe que a remoção da placa bacteriana é o principal critério a se considerar. ${ }^{11}$ É função dos cirurgiões-dentistas habilitados ao atendimento oferecerem treinamentos aos cuidadores para ensinar e esclarecer técnicas e manejos voltados para a saúde bucal das PcDs. ${ }^{7}$ As técnicas de cuidado bucal para PcD precisam ser aprofundadas, disponibilizando orientações que garantam a manutenção de uma higiene bucal satisfatória, com a instrução contínua nos serviços de saúde. ${ }^{3} \mathrm{O}$ tratamento individual, a criação de vínculos, a motivação e a competência em saúde bucal são elementos que superam as dificuldades de atendimento. $^{5}$

Preparar os profissionais desde a graduação para trabalhar com este grupo de pacientes promove sensibilização, disposição e formação técnica. ${ }^{14} \mathrm{~A}$ abordagem odontológica requer zelo, 


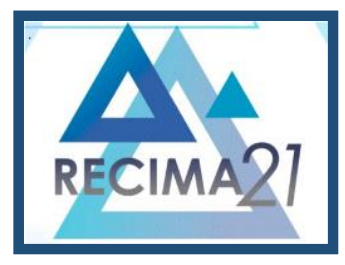

\title{
RECIMA21 - REVISTA CIENTÍFICA MULTIDISCIPLINAR
}

\author{
PESSOAS COM DEFICIÊNCIA: PERCEPÇ̃̃O DE SEUS CUIDADORES QUANTO AO \\ ATENDIMENTO ODONTOLÓGICO \\ Lauren Volquind, Lucas Bozzetti Pigozzi, Mariá Cortina Bellan, Marília Paulus, Alexandre Conde
}

paciência, estudo e determinação para evitar transtornos no consultório. Os pacientes precisam de muita atenção e ajuda para realizar e manter a higiene bucal. Cada alteração sistêmica deve ser avaliada pelos profissionais da odontologia para saber lidar com cada paciente, reconhecendo a etiologia das deficiências presentes. A avaliação pré-operatória da $\mathrm{PcD}$ é algo a se considerar antes de decidir qualquer conduta final para que seja possível proporcionar melhor qualidade de vida. Fazse mister alertar esta população de pais e responsáveis das PcDs quanto à importância de uma alimentação saudável, especificando quais os indicados para o processo de mineralização do esmalte dentário e como introduzi-los na dieta de seus filhos. A razão disso é que pessoas com deficiência estão mais vulneráveis a danos orgânicos causados pelas más condições de saúde bucal. ${ }^{25}$ Logo, quanto mais cedo é o atendimento das pessoas com deficiência, mais rápida é a ação de prevenir o aparecimento de outros problemas de maiores proporções. ${ }^{9}$

É de comum sabedoria que apresentar as dependências do consultório pode trazer mais tranquilidade para o paciente, uma vez que ele se sentirá mais ambientado. Quanto ao local, portas largas, corrimão, rampas para cadeira de rodas, salas térreas ou prédios com elevadores são adequações de acessibilidade importantes. Quando há vínculo entre a tríade (profissional-pacientefamília/cuidador), o tratamento odontológico trará bons resultados. Nas consultas periódicas, o cirurgião-dentista será o responsável por identificar anormalidades na cavidade bucal destes pacientes, sendo o mesmo também encarregado de reconhecer a necessidade de um atendimento especializado. ${ }^{9}$

A qualidade de vida da PcD depende muito de uma abordagem odontológica precoce. Infelizmente, essa não é a situação observada mundialmente. No conjunto de ações propostas para o atendimento odontológico para PcD há uma insuficiência. Isso significa que o profissional deve buscar constantemente informações e técnicas novas, incorporando-as num atendimento humanizado. Atender este público faz com que tenhamos uma postura diferente, sempre provendo interação com outros profissionais e com métodos diferenciados. ${ }^{26}$ Os encaminhamentos só devem ser feitos após a PcD ter sido avaliada para evitar que se referencie o paciente de forma descontrolada, o qual gera atraso de tratamento e ansiedade nestes indivíduos. As dificuldades mais citadas na literatura para o atendimento são a falta de treinamento, dificuldade de gerenciar os comportamentos e o grau de deficiência do paciente. ${ }^{13}$ Toda a situação do mercado de trabalho atual é um reflexo de que houve uma falha nos desempenhos curriculares dos cursos de odontologia para o preparo dos alunos para atender este grupo que cresce cada vez mais. ${ }^{27}$

\subsection{Qualidade e acesso de serviços públicos especializados em odontologia para PcD}




\title{
RECIMA21 - REVISTA CIENTÍFICA MULTIDISCIPLINAR
}

\author{
PESSOAS COM DEFICIÊNCIA: PERCEPÇ̃̃O DE SEUS CUIDADORES QUANTO AO \\ ATENDIMENTO ODONTOLÓGICO \\ Lauren Volquind, Lucas Bozzetti Pigozzi, Mariá Cortina Bellan, Marília Paulus, Alexandre Conde
}

$O$ atendimento individual e especial ainda é um desafio porque existem poucos profissionais capacitados para atender PcDs em redes públicas ou privadas. O que também desfavorece a procura por auxílio odontológico é a falta de recursos financeiros. ${ }^{5} \mathrm{O}$ tratamento desses pacientes deve conter cuidados específicos para a realidade de cada um, porém há uma deficiência de investimentos tanto pelo SUS quanto por outras instituições ao capacitar profissionais. Qualificação profissional deficiente, falta de integração na educação e na prevenção, barreiras arquitetônicas, isto é, locais de atendimento de difícil acesso, situação financeira insuficiente, medo, negligência ou desinformação são alguns fatores pelo qual impede o cirurgião-dentista de atuar de forma ideal quando se trata de pessoas com deficiência, trazendo prejuízo. ${ }^{12}$

A ausência de disciplinas com ênfase no atendimento odontológico para pessoas com deficiência na maioria dos cursos de odontologia brasileiros é o que causa as dificuldades encontradas para o tratamento em PcD atualmente na sociedade. ${ }^{28} \mathrm{~A}$ escassez de informações e a falta de habilidades independentes é o que mostra um baixo nível de higienização da PcD. ${ }^{9}$ Entretanto, a participação do cirurgião-dentista nas equipes multidisciplinares é fundamental para prática de atividades terapêuticas, preventivas e educativas na promoção da saúde bucal, melhorando o quadro geral da PcD. Quanto mais avançado o quadro de deficiência, mais a saúde bucal é comprometida e menor é o contato com o serviço odontológico. Contudo, a busca por melhorias quanto a higienização, técnicas e informações podem aumentar a expectativa de vida e saúde da PcD. ${ }^{29}$

Os cuidadores das PcDs já relataram que há um difícil acesso para atendimento porque além das barreiras sociais e financeiras, a baixa disponibilidade de cirurgiões-dentistas especializados para examinar e ver quais possíveis tratamentos, e a demora de agendamento também estão no cotidiano. ${ }^{19,27} \mathrm{O}$ desconhecimento dos serviços disponíveis, tanto de rede pública quanto privada também é apontado como fator negativo no acesso aos serviços especializados. ${ }^{19}$

O impacto da incapacidade em crianças deficientes não afeta apenas os próprios indivíduos, mas também afeta o ambiente circundante, incluindo a família e os sistemas de apoio, bem como a prestação de serviços voltados para a saúde. ${ }^{13}$ Centros de educação e saúde para PcDs são menores quando comparado a pacientes sem desvios de normalidade. ${ }^{30} \mathrm{~A}$ percepção dos cuidadores pode produzir intervenção oportuna e otimizada, além de melhorar a saúde bucal desse grupo suscetível. ${ }^{15}$ Existe uma forte necessidade de estratégias de prevenção para melhorar a higiene bucal na PcD. Conversar, distrair e usar humor são métodos utilizados pelos cuidadores para enfrentar a resistência física dos pacientes. Uma estratégia de protocolos e sessões de treinamento têm sido sugeridas para que os cuidadores se sintam mais à vontade em prestar cuidados bucais a seus pacientes e, assim, melhorar a saúde bucal. A mudança organizacional também é essencial para o sucesso e o desenvolvimento das promoções da saúde..$^{22}$ 


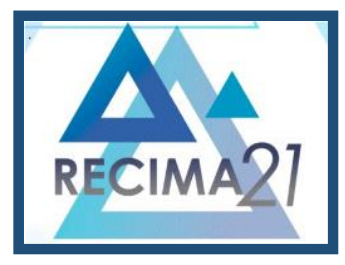

\title{
RECIMA21 - REVISTA CIENTÍFICA MULTIDISCIPLINAR
}

\author{
PESSOAS COM DEFICIÊNCIA: PERCEPÇÃO DE SEUS CUIDADORES QUANTO AO \\ ATENDIMENTO ODONTOLÓGICO \\ Lauren Volquind, Lucas Bozzetti Pigozzi, Mariá Cortina Bellan, Marília Paulus, Alexandre Conde
}

A elaboração de um sistema que atenda pessoas com deficiência, com suas complexidades e peculiaridades deve trazer interesse para o profissional, além de proporcionar promoção e prevenção de saúde, controlando doenças bucais e contribuindo uma reabilitação funcional. Atrair e capacitar os acadêmicos ao mercado de trabalho é o que mais induz a habilidade de futuramente poderem lidar com tais pacientes, diminuindo sensações negativas, colaborando na criação de valores e de um profissional integralizado. ${ }^{16}$

\section{METODOLOGIA}

\subsection{Problema da Pesquisa}

$\mathrm{O}$ atendimento e o tratamento odontológico são importantes e são um direito de todos os indivíduos de uma sociedade. A falta de profissionais especializados na área e de recursos financeiros é o que acarreta a pouca disponibilidade de serviços oferecidos para esse grupo, bem como um padrão de qualidade de atendimento inferior ao que deveria ser ofertado para eles. $\mathrm{A}$ presente pesquisa objetiva avaliar a qualidade dos atendimentos odontológicos, através da percepção dos cuidadores de pessoas com deficiência.

\subsection{Tipo da Pesquisa}

Trata-se de uma pesquisa de campo, quanti-qualitativa, transversal realizada com amostra comunitária de cuidadores de pessoas com deficiência que frequentam as dependências da clínica de rede particular VidaFisio em Caxias do Sul-RS. O presente estudo foi submetido e aprovado pelo Comitê de Ética em Pesquisa da FSG Centro Universitário sobre o parecer número 4.177.968 / CAAE 34261720.3.0000.5668.

Foram incluídos somente aqueles que aceitaram e assinaram o Termo de Consentimento Livre e Esclarecido (TCLE), concordando em fazer parte do estudo. O público-alvo foi submetido a um questionário com perguntas fechadas da pesquisa de Santos et al. (2011), com questões a respeito da qualidade de atendimento, acesso ao serviço odontológico e tratamentos especializados ofertados voltados para a pessoa com deficiência.

As perguntas que constavam no questionário proveniente do estudo de Santos et al. foram:

- Gênero do paciente;

- Idade da PcD;

- Cidade e estado em que reside;

- A receptividade no atendimento odontológico;

- O tempo de demora para o atendimento; 


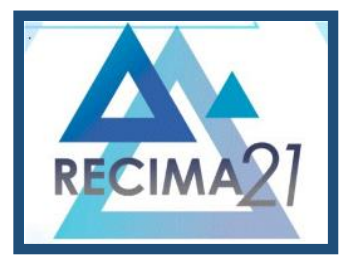

\section{RECIMA21 - REVISTA CIENTÍFICA MULTIDISCIPLINAR}

PESSOAS COM DEFICIÊNCIA: PERCEPÇÃO DE SEUS CUIDADORES QUANTO AO

ATENDIMENTO ODONTOLÓGICO

Lauren Volquind, Lucas Bozzetti Pigozzi, Mariá Cortina Bellan, Marília Paulus, Alexandre Conde

- O tempo que a PcD faz tratamento odontológico;

- O principal motivo pelo qual a PcD faz há mais de um ano o tratamento;

- A avaliação da qualidade dos atendimentos e serviços odontológicos;

- Se informações são prestadas sobre a realização dos tratamentos;

- Se foi explicado a importância de que o paciente tenha saúde bucal;

- Se houve necessidade de a PcD ser "segurada" durante o atendimento;

- O incômodo do cuidado caso a PcD tenha de ser "segurada";

- Se a PcD teve necessidade de tomar medicamento para ser atendida;

- Se foi realizado tudo aquilo que foi explicado sobre o tratamento.

Os dados coletados foram agrupados procedendo-se à análise descritiva, utilizando o programa Excel, versão 2016 (Microsoft Co., 2003). Os dados foram analisados por meio de tabelas simples, cruzadas e porcentagens. Para a análise estatística utilizou-se o software Epi Info 7.2.

\subsection{Justificativa}

A dificuldade em atender essa população gera a escassez de trabalhos científicos a respeito deste tema. Como consequência, certo despreparo profissional futuro. Este hiato trouxe motivação para desenvolver o projeto, uma vez que esse grupo está cada vez mais prevalente na nossa sociedade e a higiene bucal compromete o bem-estar das pessoas com deficiência. ${ }^{10}$

\section{RESULTADOS E DISCUSSÃo}

O presente estudo contemplou 31 entrevistados, um número obtido por amostragem de saturação que, se compararmos com outros trabalhos publicados, por exemplo, Souza et al. (20 entrevistados), Ferreira et al. (13 entrevistados), Macêdo et al. (9 entrevistados), este superou a quantidade de aplicação de questionários. Todavia, foi possível constatar, também, que outros pesquisadores aplicaram questionário em uma quantidade maior de pesquisados. $3,5,7,9,11,13,14,16,21,25,26,27,28,29$

A coleta de dados foi realizada durante o período da pandemia do COVID-19 e as pessoas com deficiência são consideradas grupo de risco. Portanto, a amostra desta pesquisa teria sido maior se tivesse ocorrido em outro período.

Conforme as orientações dadas anteriormente, foi possível perceber as adversidades encontradas pelos cuidadores quando se trata de atendimento odontológico, corroborando com experiências previamente relatadas na pesquisa de Santos et al. ${ }^{13} \mathrm{~A}$ pesquisa mostrou que grande parte das pessoas com deficiência que frequentam a clínica VidaFisio são residentes em Caxias do Sul $(96,8 \%)$, mas $3,2 \%$ residem em outra cidade. 


\section{RECIMA21 - REVISTA CIENTÍFICA MULTIDISCIPLINAR}

PESSOAS COM DEFICIÊNCIA: PERCEPÇÃO DE SEUS CUIDADORES QUANTO AO

ATENDIMENTO ODONTOLÓGICO

Lauren Volquind, Lucas Bozzetti Pigozzi, Mariá Cortina Bellan, Marília Paulus, Alexandre Conde

Sobre os dados gerais da PcD, $61,3 \%$ eram do gênero masculino e $38,7 \%$ do feminino. $O$ maior público das PcDs tinha até 20 anos de idade (74,2\%), seguido das faixas-etárias de 21-30 anos (12,9\%), 31-40 e mais de 50 anos (6,5\%), conforme Tabela 1.

Tabela 1. Descrição da amostra investigada

\begin{tabular}{llcc}
\hline Variável & Resposta & № casos & $\%$ \\
\hline Gênero & Masculino & 19 & 61,3 \\
& Feminino & 12 & 38,7 \\
\hline Idade & Até 20 & 23 & 74,2 \\
& $21-30$ & 4 & 12,9 \\
& $31-40$ & 2 & 6,5 \\
& Mais de 50 & 2 & 6,5 \\
\hline Cidade & Caxias do Sul & 30 & 96,8 \\
& Farroupilha & 1 & 3,2 \\
\hline
\end{tabular}

Quanto à receptividade na clínica odontológica em que cada PcD frequenta, 67,7\% dos entrevistados relatam que todas as pessoas são cordiais, $29 \%$ que nem todas as pessoas são e $3,2 \%$ que não foram bem recebidos. Sobre o tempo na sala de espera, $54,8 \%$ dos entrevistados relatam ser demorado e $45,2 \%$ reportam ser rápido. Quando foram questionados sobre a qualidade do atendimentos e serviços prestados em Caxias do Sul, 58,1\% dos cuidadores responderam como bom e 41,9\% como ótimo, como consta na Tabela 2. O tempo de espera, segundo a literatura, pode gerar graus de ansiedade na $\mathrm{PcD}$, o que pode acarretar a não colaboração durante os atendimentos. A boa receptividade e cordialidade também é citada na literatura com índices altos. ${ }^{10,13}$

Tabela 2. Avaliação do serviço prestado

\begin{tabular}{|c|c|c|c|}
\hline Variável & Resposta & № casos & $\%$ \\
\hline \multirow{3}{*}{$\begin{array}{l}\text { Quanto à receptividade na clínica } \\
\text { odontológica }\end{array}$} & Não fomos bem recebidos & 1 & 3,2 \\
\hline & Nem todas as pessoas são cordiais & 9 & 29,0 \\
\hline & Todas as pessoas são cordiais & 21 & 67,7 \\
\hline \multirow[t]{2}{*}{ Sobre o tempo de espera } & Pouco demorado & 17 & 54,8 \\
\hline & Rápido & 14 & 45,2 \\
\hline \multirow{2}{*}{$\begin{array}{l}\text { Como você avalia a qualidade } \\
\text { dos atendimentos e serviços } \\
\text { prestados? }\end{array}$} & Ótimo & 13 & 41,9 \\
\hline & Bom & 18 & 58,1 \\
\hline
\end{tabular}

Quanto à frequência em que a $\mathrm{PcD}$ faz tratamento, descrito na Tabela 3,41,9\% dos cuidadores responderam durar mais de dois anos e o motivo de um tempo maior de tratamento foi justificado pela PcD ser bastante difícil de manejar $(22,6 \%)$ e outras razões $(54,8 \%)$. No estudo de 


\title{
RECIMA21 - REVISTA CIENTÍFICA MULTIDISCIPLINAR
}

\author{
PESSOAS COM DEFICIÊNCIA: PERCEPÇÃO DE SEUS CUIDADORES QUANTO AO \\ ATENDIMENTO ODONTOLÓGICO \\ Lauren Volquind, Lucas Bozzetti Pigozzi, Mariá Cortina Bellan, Marília Paulus, Alexandre Conde
}

Nasiloski et al. e Macêdo et al. foi mencionada a procura tardia por tratamento odontológico, onde há mais procedimentos invasivos e mutiladores, que acabam gerando um longo tempo de tratamento, uma vez que a falta de cooperação das PcDs pode interferir também no manejo e na duração das consultas. ${ }^{7,22}$

Quando comparados com a idade, $13(56,5 \%)$ cuidadores de PcDs de até vinte anos responderam como outros motivos não definidos o motivo pelo qual fazem o tratamento há mais de um ano. Interligada ao tratamento odontológico, a manutenção preventiva é fundamental para manter a saúde bucal adequada, evitando procedimentos menos conservadores. A manutenção pode ser realizada na Unidade Básica de Saúde ou no Centro de Especialidades Odontológicas, caso a PcD não apresente comportamento satisfatório, impossibilitando seu manejo na atenção primária. ${ }^{33}$

Tabela 3. Tempo de tratamento

\begin{tabular}{llcc}
\hline Variável & Resposta & № casos & $\%$ \\
\hline Há quanto tempo, o paciente que & Primeira consulta & 4 & 12,9 \\
acompanha faz tratamento? & Menos de 1 ano & 4 & 12,9 \\
& 1 - 2 anos & 10 & 32,3 \\
& Mais de 2 anos & 13 & 41,9 \\
\hline Se você vem há mais de 1 ano, qual o & Paciente é bastante difícil & 7 & 22,6 \\
principal motivo? & Paciente tem muitos & 5 & 16,1 \\
& procedimentos para realizar & & \\
& Poucos dias de atendimento & 2 & 6,5 \\
& Outros & 17 & 54,8 \\
\hline
\end{tabular}

Quando questionados sobre a imposição da $\mathrm{PcD}$ em tomar alguma medicação para ser atendida, a maioria (87,1\%) disse não ser necessário tal atitude, assim como $64,5 \%$ dos entrevistados afirmaram que a $\mathrm{PcD}$ sob sua responsabilidade não teve de ser contida durante 0 tratamento. Todavia, aqueles que responderam que houve a necessidade de contenção durante o atendimento $(35,5 \%), 8$ entrevistados $(72,7 \%)$ declararam não se importar com a ação, pois sabiam que era necessário e $3(27,3 \%)$ reportaram que mesmo sabendo da necessidade de contenção se sentiram incomodados, como mostra na Tabela 4. Vale ressaltar que a contenção física tem se mostrado eficaz no atendimento das PcDs em consultório, pois é um método que auxilia o trabalho e o tratamento odontológico, bem como o uso de ansiolíticos, que ainda são pouco utilizados na área, mas que também trazem impacto positivo quando usados corretamente. A sedação consciente também é um método de controle comportamental, sendo possível ser realizada a nível ambulatorial ou de consultório. A sedação tem sido facilitadora ao ser realizada no atendimento odontológico. 9,13 Sendo assim, o atendimento a nível hospitalar sob uso de anestesia geral e/ou sedação venosa é considerado a última opção quando todas anteriormente já foram feitas e não bem-sucedidas, e acabaram sendo descartadas..$^{21,31}$ 


\section{RECIMA21 - REVISTA CIENTÍFICA MULTIDISCIPLINAR}

PESSOAS COM DEFICIÊNCIA: PERCEPÇ̃̃O DE SEUS CUIDADORES QUANTO AO

ATENDIMENTO ODONTOLÓGICO

Lauren Volquind, Lucas Bozzetti Pigozzi, Mariá Cortina Bellan, Marília Paulus, Alexandre Conde

Tabela 4. Sobre o atendimento

\begin{tabular}{llcc}
\hline Variável & Resposta & № casos & $\%$ \\
\hline O paciente que acompanha precisou & Sim & 3 & 9,7 \\
tomar remédio para ser atendido? & Não & 27 & 87,1 \\
& Não sei & 1 & 3,2 \\
\hline O paciente necessitou ser "segurado" & Sim & 11 & 35,5 \\
durante o tratamento? & Não & 20 & 64,5 \\
\hline Se a resposta foi sim: & Não me incomoda, pois sei que é & 8 & 72,7 \\
& necessário & 3 & 27,3 \\
& Mesmo sabendo que é & & \\
& necessário, isso me incomoda & & \\
\hline
\end{tabular}

Trinta pesquisados $(96,8 \%)$ garantiram que houve prestação de informações por parte do profissional quanto a realizações do tratamento e todos os entrevistados disseram que foi explicado a importância de uma boa saúde bucal nas pessoas com deficiência, dados apresentados na Tabela 5. Fornecer informações sobre cada etapa do tratamento tem se mostrado importante para os cuidadores usufruírem de novas habilidades e técnicas ensinadas pelo cirurgião-dentista. São as referências e as experiências que o cuidador adquire que aumentam a expectativa e a qualidade de vida da $\operatorname{PcD} .^{10,18}$ As informações sobre saúde bucal e dieta não cariogênica são importantes para manter uma adequada higiene bucal na $\mathrm{PcD}$, uma vez que este grupo tende a desenvolver mais atividades cariosas e doenças periodontais por causa da falta de destreza manual na higienização. $3,5,25,30,32$

Tabela 5. Sobre informações prestadas

\begin{tabular}{llcc}
\hline Variável & Resposta & № casos & $\%$ \\
\hline São prestadas informações sobre a & Sim & 30 & 96,8 \\
realização dos tratamentos? & Não & 1 & 3,2 \\
\hline Foi explicado por que é importante & Sim & 31 & 100,0 \\
tratar para que o paciente tenha saúde & Não & - & - \\
bucal? & & 26 & 83,9 \\
\hline Quanto ao tratamento, realizaram tudo & Sim & 5 & 16,1 \\
o que foi explicado? & Parcialmente &
\end{tabular}

Segundo Silva et al. ${ }^{3}$ a intervenção do cirurgião-dentista mostra-se importante não apenas no atendimento clínico terapêutico especializado, mas também no que diz respeito sobre a transmissão de informações fundamentais inerentes aos cuidados adaptados às PcDs, demonstrando e recomendando instrumentos facilitadores e, simultaneamente, incentivando os cuidadores acerca da importância de uma higienização oral contínua. 


\title{
RECIMA21 - REVISTA CIENTÍFICA MULTIDISCIPLINAR
}

\author{
PESSOAS COM DEFICIÊNCIA: PERCEPÇÃO DE SEUS CUIDADORES QUANTO AO \\ ATENDIMENTO ODONTOLÓGICO \\ Lauren Volquind, Lucas Bozzetti Pigozzi, Mariá Cortina Bellan, Marília Paulus, Alexandre Conde
}

Após o programa "Brasil Sorridente", o acesso aos serviços de saúde bucal no setor público aumentou, mas ainda não foi suficiente para atender a grande demanda de $\operatorname{PcD}$. Por conseguinte, 0 setor privado também é buscado para suprir as necessidades odontológicas. ${ }^{33}$ Os sistemas de saúde público e privado possuem diferentes abordagens. Embora estejam relacionados, cabe às condições financeiras da família da PcD e ao acesso de serviço para que todos os âmbitos destes setores sejam usufruídos. ${ }^{34}$ Os resultados dos estudos de Cardoso et al. mostraram que $50 \%$ dos atendimentos odontológicos são feitos no setor público, apresentando um equilíbrio de atividade terapêutica entre rede pública e privada.

Há uma grande necessidade de programas preventivos e de controle no setor público, bem como uma oferta maior de cirurgiões-dentistas para estas pessoas. ${ }^{35} \mathrm{~A}$ possível justificativa para esta falta de profissionais se deve à formação acadêmica e à disposição que eles apresentam perante o atendimento de pessoas com deficiência. ${ }^{10,14,15,16,17,35} \mathrm{O}$ acesso à saúde é um direito de todos, e o cirurgião-dentista habilitado acaba trazendo conhecimento e incentivos necessários para que 0 tratamento odontológico obtenha sucesso, sempre priorizando o bem-estar do paciente..$^{35,36}$

Em outras palavras, para a concretização dos direitos à saúde deste grupo, garantidos na Constituição Federal, faz-se necessário a articulação das políticas públicas voltadas para pessoas com deficiência, por meio de programas específicos, de caráter multidisciplinar que resultem em ações e atividades voltadas para a atenção integral que tenham por objetivo a inclusão social e melhoria da qualidade de vida destes cidadãos.

\section{CONCLUSÃO}

Na presente pesquisa foi possível identificar a existência de conhecimento dos cuidadores a respeito do tratamento odontológico realizado na $\operatorname{PcD}(96,8 \%)$, mostrando a importância de informações sobre uma saúde bucal adequada na vida dessas pessoas. Além disso, a existência de acesso ao serviço odontológico para o grupo pesquisado na cidade $(99,9 \%)$ também foi constatada. Entretanto, os resultados apresentados neste estudo em relação a qualidade de atendimento odontológico não podem afirmar que existe satisfação por parte dos cuidadores, devido ao pequeno número da amostra. Portanto, sugere-se uma nova pesquisa, com um número amostral mais amplo para que seja possível avaliar melhorias para o atendimento das PcDs.

\section{REFERÊNCIAS}

1. Lima DC, Saliba NA, Garbin AJl, Fernandes LA, Garbin CAS. A importância da saúde bucal na ótica de pacientes hospitalizados. Ciênc. Saúde Colet. ABRASCO - Assoc. Brasil Saúde Colet. 2011; 16(1):1173-80.

2. Instituto Brasileiro de Geografia e Estatística [homepage na internet]. Censo Demográfico: População residente por tipo de deficiência permanente, 2010. [acesso em 28 mar 2020]. Disponível 


\title{
RECIMA21 - REVISTA CIENTÍFICA MULTIDISCIPLINAR
}

\author{
PESSOAS COM DEFICIÊNCIA: PERCEPÇÃO DE SEUS CUIDADORES QUANTO AO \\ ATENDIMENTO ODONTOLÓGICO \\ Lauren Volquind, Lucas Bozzetti Pigozzi, Mariá Cortina Bellan, Marília Paulus, Alexandre Conde
}

em:

https://www.ibge.gov.br/estatisticas/sociais/populacao/9662-censo-

demografico2010.html?edicao $=9749 \& \mathrm{t}=$ destaques

3. Silva HM, Oliveira KB de, Silva RV, Coelho PM. A percepção dos cuidadores de Pacientes com Necessidades Especiais na higiene bucal em um município baiano. Rev Faculdade Odontol Lins. 2018; 28(1):27-39.

4. Brasil. Ministério da Saúde. Secretaria de Atenção à Saúde. Departamento de Atenção Básica. Saúde Bucal / Ministério da Saúde, Secretaria de Atenção à Saúde, Departamento de Atenção Básica. - Brasília: Ministério da Saúde, 2008. [acesso em 28 mar 2020]. Disponível em: http://bvsms.saude.gov.br/bvs/publicacoes/saude bucal.pdf

5. Queiroz FS, Rodrigues MMLF, Cordeiro Junior GA, Oliveira AB, Oliveira JD de, Almeida ER de. Avaliação das condições de saúde bucal de Portadores de Necessidades Especiais. Rev Odontol UNESP. 2014; 43(6):396-401.

6. Nasiloski KS, Silbeira ER da, César Neto JB, Schardosim LR. Avaliação das condições periodontais e de higiene bucal em escolares com transtornos neuropsicomotores. Rev Odontol UNESP. 2015; 44(2):103-107.

7. Martins RB, Andia-Merlin R, Giovani EM. Avaliação sobre a atenção com a saúde bucal de pacientes com necessidades especiais. J Health Sciences Institute. 2013; 31(4):360-367.

8. Oliveira ALBM, Giro EMA. Importância da Abordagem Precoce no Tratamento Odontológico de Pacientes com Necessidades Especiais. Odonto. 2011; 19(38):45-51.

9. Portolan C, Velaski D, Maçalai M, Hochmuller M, Cezar M, Portella V. Odontologia e Pacientes Especiais: Conhecer, Orientar e Prevenir. Rev Saúde Integrada. 2017; 10(20):7-15.

10. Gonçalves JB. Atendimento odontológico à pacientes com necessidades especiais: uma revisão de literatura [monografia]. Conselheiro Lafaiete: Universidade Federal de Minas Gerais - UFMG; 2012.

11. Soares J, Volpato LER, Castro PHS, Lambert NA, Borges AH, Carvalhosa AA de. Avaliação do conhecimento sobre saúde bucal de pais e cuidadores de crianças e adolescentes com deficiência. J Health Sci Inst. 2013; 31(3):239-243.

12. Spezzia S, Bertolini SR. Ensino odontológico para pacientes especiais e gestão em saúde. J Oral Invest. 2017; 6(1):85-98.

13. Santos FS dos, Camargo JF, Martins AT, Scannavino FLF, Rodrigues R. Percepção dos cuidadores sobre os aspectos éticos do atendimento odontológico a pacientes com necessidades especiais. Odontol. Clín. -Cient. 2011; 10(4):337-340.

14. Jacomine JC, Ferreira R, Sant'anna ACP, Rezende MLR de, Greghi SLA, Damante CA, et al. Saúde bucal e Pacientes com Necessidades Especiais: percepções de graduandos em Odontologia da FOB-USP. Rev ABENO. 2018; 18(2):45-54

15. Andrade APP de, Eleutério ASL. Pacientes portadores de necessidades especiais: abordagem odontológica e anestesia geral. Rev. Bras. Odonto. 2015; 72(1):66-69.

16. Cruz AOS, Cavalcante MLT de H, Labuto MM. Limitações do Cirurgião-Dentista na Abordagem Clínica no Atendimento de Pacientes com Necessidades Especiais. Cad Odontol do Unifeso. 2020; $1(2): 44-69$.

17. Ferreira SH, Suita RA, Rodrigues PH, Kramer PF. Percepção de estudantes de graduação em Odontologia frente ao atendimento de pessoas com deficiência. Rev ABENO. 2017; 17(1):87-96. 


\title{
RECIMA21 - REVISTA CIENTÍFICA MULTIDISCIPLINAR
}

\author{
PESSOAS COM DEFICIÊNCIA: PERCEPÇÃO DE SEUS CUIDADORES QUANTO AO \\ ATENDIMENTO ODONTOLÓGICO \\ Lauren Volquind, Lucas Bozzetti Pigozzi, Mariá Cortina Bellan, Marília Paulus, Alexandre Conde
}

18. Brasil. Presidência da República. Secretaria Nacional de Promoção dos Direitos da Pessoa com Deficiência. Secretaria de Direitos Humanos da Presidência da República. Avanços das Políticas Públicas para as Pessoas com Deficiência. Distribuição gratuita - Uma análise a partir das Conferências Nacionais. 1ํㅡㄹ edição, Brasília; 2012.

19. Silva RBS. Acesso aos serviços odontológicos de saúde para pacientes especiais: revisão sistemática [monografia]. Lagarto: Universidade Federal de Sergipe - UFS; 2018.

20. Penha ES, Tenório DA, Fonseca FRA, Guênes GMT, Montagna E. Caracterização do componente curricular Odontologia para Pacientes com Necessidades Especiais nos cursos de Odontologia do estado da Paraíba. Rev ABENO. 2018; 18(2):13-19.

21. Pereira CM, Castro CEB, Rocha GROM da, Sá PFG de. Importância do conhecimento sobre saúde bucal dos cuidadores de pacientes com necessidades especiais. Rev Faculdade Odontol Lins. 2019; 29(1):3-12.

22. Macêdo GL, Lucena EES, Lopes IKR, Batista LTO. Acesso ao Atendimento Odontológico dos Pacientes Especiais: A Percepção de Cirurgiões-Dentistas da Atenção Básica. Rev Ciênc Plural. 2018; 4(1):67-80.

23. Lim GXD. Special care dentistry in a charity clinic: Demographic analysis and barriers to care in Singapore. Tzu Chi Med J. 2019; 31(4):232-39.

24. Souza LV, Abrão LSO, Loureiro RMT, Castro AM de, Oliveira FS de. Percepção dos pais sobre a qualidade de vida relacionada à saúde bucal de crianças com paralisia cerebral. Hor Ci. 2010; 4(2):129.

25. Nqcobo C, Ralephenya T, Kolisa YM, Esan T, Yengopal V. Caregivers' perceptions of the oralhealth-related quality of life of children with special needs in Johannesburg, South Africa. Health Sa Gesondheid. 2019; 24(1056):1-7.

26. Rajan S, Kuriakose S, Varghese BJ, Asharaf F, Suprakasam S, Sreedevi A. Knowledge, Attitude, and Practices of Dental Practitioners in Thiruvananthapuram on Oral Health Care for Children with Special Needs. Int J Clin Pediatr Dent. 2019; 12(4):251-54.

27. Cho SM, Song J, Chang J. Differences between caregiver-perceived and dentist-assessed oral health status of patients among intellectual disabilities. Community Dent Health. 2019; 4(36):255-61.

28. Phlypo I, Palmers E, Janssens L, Marks L, Jacquet W, Declerck D. The perception of oral health and oral care needs, barriers and current practices as perceived by managers and caregivers in organizations for people with disabilities in Flanders, Belgium. Clin Oral Invest. 2019; 10(1):1-10.

29. Crescêncio MCC, Cristiano DP, Simões PW, Sonego FGF. Análise do Conhecimento de Pais ou Responsáveis sobre a Saúde Bucal dos Filhos com Necessidades Especiais. Rev. Odontol. Univ. Cid. São Paulo. 2018; 30(2):144-56.

30. Ningrum V, Wang W, Liao H, Bakar A, Shih Y. A special needs dentistry study of institutionalized individuals with intellectual disability in West Sumatra Indonesia. Sci Rep. 2020; 10(153):1-8.

31. Marchioni SAE. Investigação sobre o uso do condicionamento pelos alunos de odontologia no atendimento a deficientes mentais. Infanto Rev Neuropsiquiatr Infanc Adolesc. 1998; 6(3):127-33. 


\section{RECIMA21 - REVISTA CIENTÍFICA MULTIDISCIPLINAR}

PESSOAS COM DEFICIÊNCIA: PERCEPÇ̃̃O DE SEUS CUIDADORES QUANTO AO

ATENDIMENTO ODONTOLÓGICO

Lauren Volquind, Lucas Bozzetti Pigozzi, Mariá Cortina Bellan, Marília Paulus, Alexandre Conde

32. Moreira TS, Ferreira RB, Vieira LDS. Nutrição do paciente TEA relacionado a doença cárie [monografia]. Brasília: Centro Universitário do Planalto Central Apparecido dos Santos UNICEPLAC; 2019.

33. Ortega MM, Saliba TA, Garbin AJI, Garbin CAS. Assistência em saúde bucal na percepção das pessoas com deficiência visual. Cad. Saúde Colet. 2019; 27(3):331-337.

34. Condessa AM. Acesso das pessoas com deficiência aos serviços de saúde bucal no Brasil [monografia]. Porto Alegre: Universidade Federal do Rio Grande do Sul - UFRGS; 2019.

35. Cardoso AMR, Brito DBA, Alves VF, Padilha WWN. O acesso ao cuidado em saúde bucal para crianças com deficiência motora: perspectivas dos cuidadores. Pesq Bras Odontoped Clin Integr. $2011 ; 11(4): 593-599$

36. Freire ALASS. Saúde bucal para pacientes com necessidades especiais: análise da implementação de uma experiência local [tese]. Rio de Janeiro: Escola Nacional de Saúde Pública Sérgio Arouca; 2011. 